\title{
PREDICCIÓN DE FLUJO MULTIFÁSICO EN SISTEMAS DE RECOLECCIÓN DE CRUDO: DESCRIPCIÓN DE REQUERIMIENTOS
}

\author{
Gustavo Andrés Valle Tamayo'; Felipe Romero Consuegra' ${ }^{1}$; Manuel Enrique Cabarcas Simancas ${ }^{2 *}$ \\ ${ }^{1}$ Petroleum Consulting Company. Cra. 29 \# 16 - 36 Int. 301. Bucaramanga. Colombia. \\ ${ }^{2}$ Universidad Industrial de Santander (UIS). Carrera 27 Cll 9, Ciudad Universitaria. Colombia. \\ *E-mail: cabarcas@uis.edu.co
}

\section{RESUMEN}

\begin{abstract}
Durante los últimos 60 años numerosos autores se han dedicado al estudio del comportamiento de flujo multifásico en tuberías. En la literatura existe un extenso número de correlaciones y modelos para la predicción y evaluación de gradientes de presión, patrones de flujo, holdup de líquido, y propiedades del fluido. El cálculo fiable de escenarios a partir de un modelo detallado y riguroso, garantiza el éxito de la operación. La toma de decisiones sin un conocimiento previo de los diversos modelos involucrados en la predicción del comportamiento de flujo, ocasiona estimaciones inadecuadas, y gastos innecesarios en recurso humano y de cómputo. Esto ocurre en muchas ocasiones debido a la falta de conocimiento de los parámetros que afectan los modelos hidráulicos, y a la inexistencia de una metodología que modele estos perfiles; con el fin de posteriormente poder predecir su comportamiento de forma acertada. En este trabajo se presenta una revisión de los métodos más relevantes en la industria del petróleo para caracterizar el flujo multifásico. Además, se propone una metodología general para el uso apropiado de los diversos simuladores en estado estable que existen comercialmente.
\end{abstract}

Palabras Clave: Aseguramiento de Flujo, Flujo Multifásico, estado estable.

\section{MULTI-PHASE FLOW PREDICTION IN CRUDE COLLECTION SYSTEMS: DESCRIPTION OF REQUIREMENTS}

\begin{abstract}
For the last 60 years, many authors have studied the behavior of multiphase flow in pipelines. An extensive number of correlations and models exist in the literature for the evaluation and prediction of pressure gradients, flow patterns, liquid holdup, and fluid properties. The reliable calculation of scenarios from a detailed and rigorous model, guarantees the success of the operation. Decision making without prior knowledge of the various models involved in the prediction of flow behavior, leads to inadequate estimates, and unnecessary expenditures on human resources and computation. This happens in many occasions due to the lack of knowledge regarding the parameters affecting the hydraulic models, and to the lack of a methodology to model these profiles, which allows proposing situations that respond to the events for their respective prediction. This paper presents a review of the most relevant methods in the petroleum industry to characterize multiphase flow. A general methodology for an appropriate use of the various commercially available steady-state simulators is also proposed.
\end{abstract}

Key Words: Flow Assurance, Multiphasic Flow, Stable state.

Cita: Valle, G., Romero, F. y Cabarcas, M. (2017). Predicción de flujo multifásico en sistemas de recolección de crudo: descripción de requerimentos. Revista Fuentes: El reventón energético, 15 (1), 87-99. 


\section{INTRODUCCIÓN}

Un tema persistente a lo largo del estudio del flujo multifásico ha sido la necesidad de modelar y predecir el comportamiento, y los fenómenos que estos manifiestan. La complejidad de los sistemas de producción, la variación de las propiedades de los fluidos (i.e. líquido, gas) en función de la presión y la temperatura, y la existencia de una diversidad de correlaciones para el cálculo de características hidráulicas, ocasionan que el diseño de sistemas multifásicos sea complejo para la producción ingenieril.

Durante más de un siglo, la industria petrolera ha enfocado esfuerzos para caracterizar el flujo multifásico, sin embargo esta caracterización a través de modelados matemáticos rigurosos, se ha empleado desde hace sólo 60 años. Por lo cual, en las últimas décadas los ingenieros han recurrido cada vez más a herramientas computacionales de simulación para modelar el flujo multifásico, contemplando una variedad de aplicaciones concernientes al aseguramiento de flujo (Shippen y Bailey, 2012).

En la actualidad, los modelos de flujo multifásico en estado estacionario y transitorio están firmemente adscritos a herramientas de simulación, facilitando el estudio del comportamiento del sistema de producción desde el yacimiento hasta el separador. Sin embargo, uno de los grandes retos para la descripción de estos procesos, es realizar una especificación adecuada de los requerimientos que conlleven al desarrollo de la caracterización del flujo de manera exitosa (Pressman, 2009). Por tanto, en busca de asegurar el proceso de manera metódica y sistemática, esta investigación se centra en los parámetros necesarios que garanticen una descripción apropiada del flujo en dos fases (gas y líquido) en estado estacionario, constituida por una estructura enfocada al modelado; para el cual existen una gran diversidad de correlaciones y enfoques en la literatura. Si bien la presencia de sólidos, fluidos complejos y flujo con cuatro (o más) fases sí existe; tales flujos están más allá del alcance de este trabajo.

\section{MODELADO DEL FLUJO PARA SU ASEGURAMIENTO}

El diseño a gran escala de tuberías y facilidades involucra garantizar la consecución de los objetivos de producción mediante la predicción, prevención, y solución de los problemas originados por el comportamiento de las sustancias transportadas (i.e. gases, líquidos y sólidos, ya sea separados o en condiciones de fases múltiples) (Di Lullo, 2012). Típicamente el aseguramiento de flujo es discutido en términos de las características de los fluidos de producción y las soluciones ingenieriles, para afrontar los retos originados a razón del fluido. La comprensión de estos criterios, y el desarrollo de simuladores computacionales han permitido un avance significativo en el área de diseño ingenieril. Por lo tanto, la ciencia es fundamental para la simulación, la simulación es esencial para la ingeniería y la ingeniería es imprescindible para la ejecución y operación.

Existe una gran variedad de herramientas de modelado de flujo en tuberías que están disponibles comercialmente, pero con el fin de lograr un uso correcto y eficiente de ellas, se requiere comprender: i) ¿qué ejecutan los programas?, ii) ¿cómo trabajan?, y, iii) ¿cuáles son sus limitaciones? Sin embargo los estados del arte de las herramientas computacionales que se implementan actualmente (Bratland, 2008) no son lo suficientemente adecuados para ser fiables, a razón de ser licenciados bajo el seudónimo de privativos. Debido a lo anterior, ellos son tratados como "cajas negras", permitiendo sólo una vaga comprensión de cómo funcionan. De tal manera, existe el peligro de que el aprendizaje de cómo simular, pueda ser interpretado como la necesidad de aprender a interactuar con el programa de simulación; cuando el interés debería centrarse en el entendimiento de las posibilidades y limitaciones del programa, y cómo interpretar y comprobar los resultados. En la Tabla 1 se resume un listado de simuladores para flujo multifásico en estado estable.

La complejidad del cálculo de flujo en la tubería depende del fluido que se transporte y de los fenómenos que se deseen investigar. En la Figura 1 se evidencian los diferentes parámetros que afectan la complejidad de estos procesos, conformando los elementos que componen un simulador de flujo.

A continuación se procederá a describir en detalle los componentes característicos de un simulador en estado estable para redes de recolección de flujo multifásico, considerando efectos térmicos (i.e. leyes de calor). 
Predicción de flujo multifásico en sistemas de recolección de crudo: descripción de requerimentos

Tabla 1. Simuladores para flujo multifásico en estado estable. Modificado de (Bratland, 2008)

\begin{tabular}{|c|c|c|}
\hline Nombre & Empresa Contacto & Comentarios \\
\hline AMATHYSTE & $\begin{array}{l}\text { KAPPA } \\
\text { www.kappaeng.com }\end{array}$ & $\begin{array}{l}\text { Módulo dedicado al análisis del desempeño de los pozos (i.e. IPR, VLP). En capacidad } \\
\text { para modelar flujo en } 2 \text { y } 3 \text { fases. }\end{array}$ \\
\hline DPDL & $\begin{array}{l}\text { University of Tulsa } \\
\text { www.tuffp.utulsa.edu }\end{array}$ & $\begin{array}{l}\text { Este software es integrado con PIPEPHASE y PIPESIM, pero también es usado } \\
\text { separadamente. En capacidad para modelar flujo en } 2 \text { y } 3 \text { fases. }\end{array}$ \\
\hline $\begin{array}{l}\text { HYSYS Pipe } \\
\text { Segement }\end{array}$ & $\begin{array}{l}\text { AspenTech } \\
\text { Aspentech.com }\end{array}$ & $\begin{array}{l}\text { No es un modelo robusto. AspenTech recomienda otras herramientas software para } \\
\text { realizar análisis complejos. }\end{array}$ \\
\hline HYSYS PEPESYS & $\begin{array}{l}\text { AspenTech } \\
\text { Aspentech.com }\end{array}$ & $\begin{array}{l}\text { Módulo licenciado separadamente de los paquetes de simulación de Hysys. Más avanzado } \\
\text { que Hysys Pipe Segment y usado para diseño y análisis de sistema de recolección. }\end{array}$ \\
\hline PIPESIM & $\begin{array}{l}\text { Schlumberger } \\
\text { www.slb.com }\end{array}$ & $\begin{array}{l}\text { Uno de los simuladores más conocidos y usados para el modelado del flujo multifásico. } \\
\text { Desarrollado para tener compatibilidad e integración con el simulador Eclipse. }\end{array}$ \\
\hline GAP & $\begin{array}{l}\text { Petroleum Experts } \\
\text { Petex.com }\end{array}$ & $\begin{array}{l}\text { Hace parte del paquete de modelado Integral de producción de Petroleum Experts. En } \\
\text { capacidad para modelar flujo en } 2 \text { y } 3 \text { fases. }\end{array}$ \\
\hline PROFES & $\begin{array}{l}\text { AspenTech } \\
\text { Aspentech.com }\end{array}$ & $\begin{array}{l}\text { Modelos dinámicos para flujo multifásico implementados en el entorno de Aspen } \\
\text { HYSYS. En capacidad para modelar flujo en } 2 \text { y } 3 \text { fases. También, puede realizar análisis } \\
\text { en estado transiente, al incluir el modulo Profes Transient. }\end{array}$ \\
\hline PIPEPHASE & $\begin{array}{l}\text { Simsci-Esscor (Desarrollado } \\
\text { actualmente por Invensys) } \\
\text { www.simsci-esscor.com }\end{array}$ & $\begin{array}{l}\text { Desarrollado para simulaciones de pozos y redes complejas. En capacidad para modelar } \\
\text { flujo en } 2 \text { y } 3 \text { fases. Puede vincularse como un módulo de la herramienta TACITE } \\
\text { transient. }\end{array}$ \\
\hline PIPEFLO & $\begin{array}{l}\text { Neotechnology Consultants Ltd. } \\
\text { Neotec.com }\end{array}$ & $\begin{array}{l}\text { Uno de los más antiguos simuladores en estado estable del flujo multifásico. En capacidad } \\
\text { para modelar flujo en } 2 \text { fases. }\end{array}$ \\
\hline TUFF Pro & $\begin{array}{l}\text { University of Tulsa } \\
\text { www.tuffp.utulsa.edu }\end{array}$ & $\begin{array}{l}\text { Este software es integrado en PIPEPHASE y PIPESIM, pero también es usado } \\
\text { separadamente. En capacidad para modelar flujo en } 2 \text { y } 3 \text { fases. }\end{array}$ \\
\hline
\end{tabular}

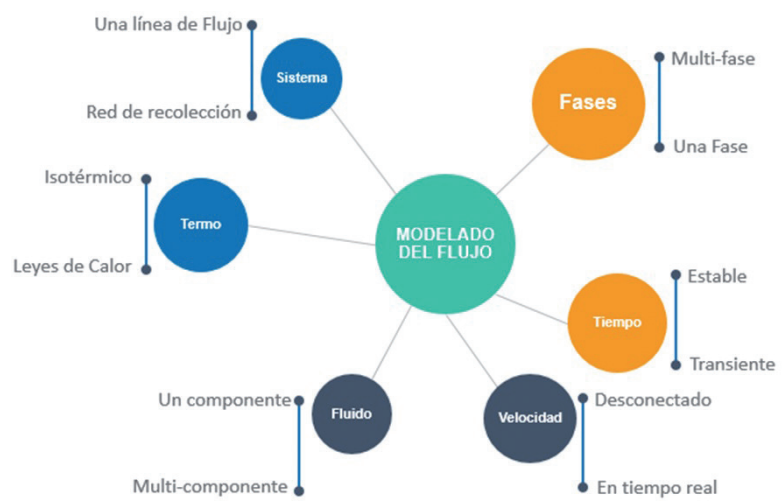

Figura 1. Parámetros que afectan la complejidad del modelado del flujo en tubería

\section{PERSPECTIVA DEL FLUJO MULTIFÁSICO EN ESTADO ESTABLE}

En 1992, Brill y Arirachakaran presentaron una clasificación para catalogar los modelos de flujo multifasico atendiendo criterios de: i) complejidad fisica, ii) "aplicación ingenieril", y, iii) etapas cronologicas respecto a la evolución investigativa. Esta tipificación fue complementada posteriormente por las investigaciones de Shippen y Bailey (2012), Ahmed y Mohammed (2014), y, Jerez-Carrizales et al. (2015).
En la Figura 2 se muestra la evolución del modelado del flujo multifásico, evidenciando una selección de los modelos más implementados en la literatura. La línea cronológica (eje de las abscisas) está asociada a dos criterios: Evolución investigativa (parte superior), y aplicación ingenieril (parte inferior). El eje vertical de la misma, ilustra el desarrollo histórico, y la tendencia en general de estos a adoptar una mayor complejidad física. El deseo de generalizar los modelos a través de condiciones más amplias, se refleja en el número de fases, y en el rango de ángulos de inclinación que se pueden considerar para el modelado. Otros aspectos de la generalización no capturados en esta figura, incluyen la evolución de los modelos para escalar mayores diámetros de tuberías, y hacer frente a rangos más amplios de propiedades de fluidos tales como la viscosidad. Por lo cual, el eje de la ordena se subdivide en 5 categorías generales:

- Modelos Monofásicos-homogéneos: Sin deslizamiento, sin patrón de flujo (Darcy, 1857; Weisbach, 1845; Moody, 1944). El factor de fricción se basa únicamente en el número de Reynolds de la mezcla (Reynolds, 1883), considerando propiedades promedio en función del volumen para las fases, tales como densidad y viscosidad (Rott, 1990). Éste enfoque generalmente no se 
implementa en la práctica, excepto con simuladores monofásicos para proporcionar una aproximación rápida de efectos de flujo multifásico (a menudo con factores de ajuste). Además, pueden utilizarse para comparar las mejoras que se obtienen, al considerar los efectos de deslizamiento de modelos multifásicos más refinados.

- Modelos Empíricos, categoría "A": Sin deslizamiento, sin patrón de flujo. La densidad de la mezcla es calculada por medio de la relación gas/líquido. Se asume que las fases se mueven a una misma velocidad. Estas correlaciones ajustan el factor de fricción asociado a mezclas de dos fases sin deslizamiento, por lo cual, se limitan a un conjunto acotado de datos experimentales y / o de campo. No realizan distinciones de patrones de flujo (Lockhart y Martinelli, 1949; Poettmann y Carpenter, 1952; Baxendell y Thomas, 1961).

- Modelos Empíricos, categoría "B": El deslizamiento es considerado; a diferencia del patrón de flujo. Se requiere una correlación tanto para el cálculo del hold up de líquido como para el factor de fricción. Se provee de un método para predecir la fracción de tubería ocupada por cada fase. El líquido y el gas pueden viajar a diferentes velocidades. Se implementan las mismas correlaciones para determinar el hold up de líquido y los patrones de flujo (Drift Flux -Zuber y Findlay, 1965; Holmes, 1977-; Flanigan, 1958; Hagedorn y Brown, 1965; Eaton et al., 1967; Dukler, 1969; Gray, 1974).

- Modelos Empíricos, categoría "C": Se considera el deslizamiento entre fases y los patrones de flujo. Se utilizan correlaciones para predecir el holdup de líquido y el factor de fricción, así como métodos para predecir los patrones de flujo (Duns y Ros, 1963; Orkiszewski, 1967; Beggs y Brill, 1973; Mukherjee y Brill, 1985; SLB Drift flux-Shi et al., 2005- ).

- Modelos Mecanísticos (Fenomenológicos): Se denominan a veces modelos "de dos fluidos" o "multifluidos" (Aziz et al., 1972; Taitel y Dukler, 1976; Hasan y Kabir, 1988; Xiao et al., 1994, Ansari et al., 1994; Petalas y Aziz, 2000; TUFFP Unified - Petalas et al., 2003-; OLGA -Bendiksen et al., 1991, LEDAflow -Danielson et al., 2005- ). Estos modelos resuelven las ecuaciones de momentum para cada fase de la mezcla. La continuidad es preservada mediante balances de masa simultáneos en los fluidos. Los patrones de flujo y sus criterios de transición se solucionan de manera explícita o implícitamente. Los modelos varían según el grado de empirismo utilizado para formular sus relaciones de proximidad al comportamiento real del fluido, $y$, como tales, representan un espectro de complejidad (e.g. diámetro de la gota de aceite, diámetro de la burbuja de gas) (Ellul, et al., 2004). Por ejemplo, el modelo de Fernández et al. (1983), incluye 22 ecuaciones simultáneas que describen en detalle el comportamiento hidrodinámico de los tapones en las tuberías verticales. E incluso un modelo tan refinado como éste, todavía, posee cierto empirismo para realizar una aproximación. Por otro lado, otros modelos de este tipo -e.g. Xiao y Shoup (1998) y Ansari et al. (1994)- tienen una mayor dependencia de las relaciones empíricas.

Los autores identificaron tres (3) periodos evolutivos, categorizando cada una de las correlaciones anteriormente descritas, de acuerdo a la comprensión de los fenomenos y recursos para el desarrollo de los mismo:

- "Periodo empírico" (1950-1975): Los modelos fueron desarrollados en base al ajuste de datos experimentales y / o de campo, considerando el flujo como una mezcla homogénea; estos modelos son limitados en términos de precisión y amplitud de aplicación.

- "Periodo del despertar" (1975-1985): En respuesta a la creciente comprensión de las deficiencias del enfoque empírico, la investigación comenzó a centrarse en un tratamiento más fundamental de la física subyacente. Durante estos años, empezó el auge de la implementación de computadores personales por parte de las compañías, para predecir la distribución de caudales y presión en las tuberías.

- "Periodo del modelado" (1980-presente): La era actual ha visto la aparición de modelos multifluidos más generalizados, basados en ecuaciones combinadas de momentum y transiciones de régimen de flujo, los cuales incorporan la física fundamental (i.e. equilibrios de fuerzas y análisis de estabilidad). En general los modelos desarrollados en ésta época son más exactos a los que le preceden; no obstante, muchas de las relaciones de proximidad siguen siendo de naturaleza empírica. Debido a lo anterior, nuevas herramientas computacionales han sido creadas para resolver estas ecuaciones.

Asimismo, en la Figura 2 se observa una segunda escala en relación a la "Aplicación ingenieril", que manifiesta 
los avances en los métodos aplicados a las soluciones de ingeniería de flujo multifásico, es decir,

- Intuición: No había soluciones rigurosas disponibles. La experiencia y la "intuición de la ingeniería” eran la base de las soluciones ingenieriles.

- Gráficos: La comprensión mejorada de las correlaciones, dio lugar a métodos gráficos, tales como nomogramas para ayudar a solucionar algunos problemas de flujo multifásico.

- Estado Estable: Con la llegada de un sistema de cómputo más accesible, y una mejor comprensión fenomenológica, se aplicó el análisis en estado estacionario al modelado de pozos (NODAL), tuberías y, eventualmente, redes complejas de gran tamaño. Esta solución ganó una rápida aceptación en la industria, y representó un cambio en la capacidad de modelar el comportamiento del sistema con mayor exactitud y rapidez.

- Estado Transiente: Este tipo de soluciones se tomaron en cuenta a finales de los años ochenta, basándose en gran medida en los avances en la industria nuclear; fundamentada en la necesidad de modelar acontecimientos transitorios como el apagado y puesta en marcha de procesos, y tapones inducidos por el terreno.

- Acoplado: Hacia mediados de la década de 1990, se realizaron esfuerzos para acoplar la simulación de yacimiento, a los modelos de pozos y redes. Consecuentemente, se amplió para considerar redes de recolección más complejas, instalaciones de procesos, tuberías de exportación y, finalmente, fiscalización.

Los enfoques presentados, capturan los modelos en una forma suficientemente completa para usarse en aplicaciones prácticas de modelado de pozos y tuberías (y, por lo tanto, son más reconocibles para el ingeniero); sin embargo, algunas de las contribuciones claves en los últimos años, han sido las relaciones de aproximación que están involucrados en la estructura moderna de la modelización mecanística. Esta área también ha evolucionado para acomodar amplias gamas de condiciones de flujo, dirigiéndose hacia un tratamiento más fundamental de los mecanismos físicos básicos para caracterizarlo. Shoham (2006) proporciona un estudio detallado de estas relaciones para los modelos mecanístico.

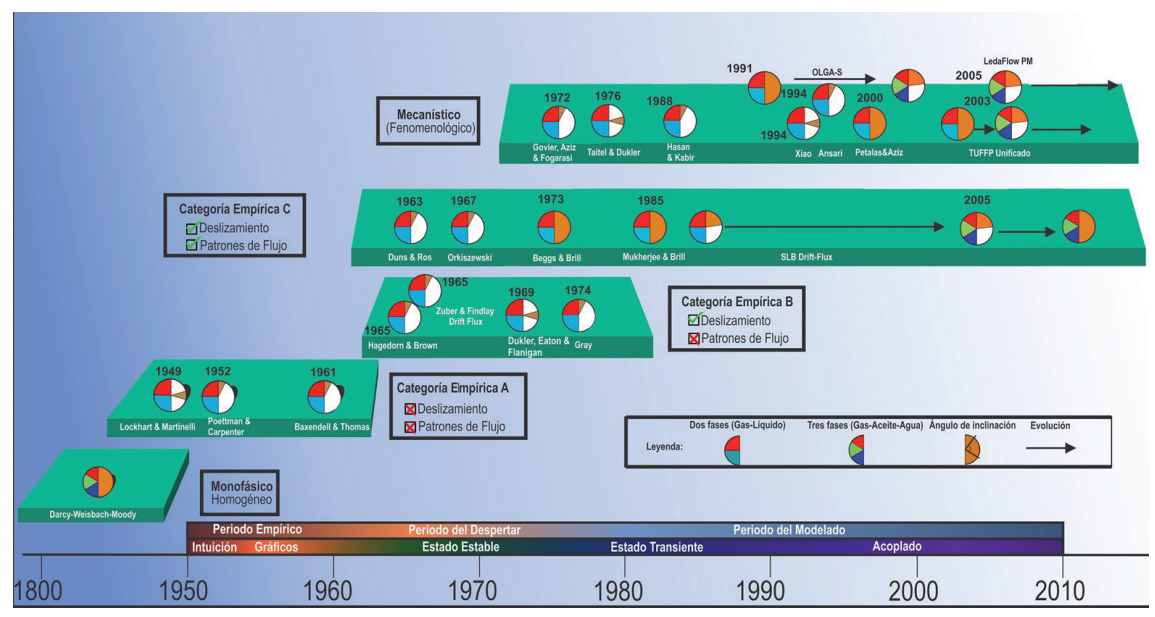

Figura 2. Evolución del modelado del flujo multifásico

\section{DESEMPEÑO DE LAS CORRELACIONES DE FLUJO}

En la actualidad son comunes las herramientas de simulación de flujo multifásico en estado estacionario; sin embargo, la gran mayoría de operadores de estos simuladores, tienen un vago conocimiento referente a esta temática; y al tener que seleccionar uno de los muchos modelos habilitados en el simulador, la tarea se vuelve desalentadora. Aquellos operadores que no aprecian el contexto histórico, las limitaciones y la aplicabilidad de las diversas opciones, están justificadamente tentados a elegir un modelo considerado como el más universalmente aplicable, el cuál generalmente es uno con una percepción (correcta o no) de alto costo de desarrollo.

A continuación se enunciarán estudios en la literatura relacionados con la evaluación de diferentes métodos de predicción de la caída de presión, sin contemplar 
aquellos modelos mecanístico desarrollados por empresas comerciales (i.e. OLGAS, TUFFP, LedaFlow). Los autores a mencionar, contrastaron los pronósticos de gradientes de presión obtenidos, con valores teóricos derivados de mediciones de datos en campo. El propósito de esta revisión literaria, es determinar la precisión de los diversos modelos de predicción de caída de presión, para orientar al ingeniero de producción de petróleo en la selección de las correlaciones de flujo multifásico.

Por lo tanto, de acuerdo a la orientación de flujo (i.e. vertical $\mathrm{u}$ horizontal), se tienen los siguientes estudios comparativos:

\section{COMPARACIÓN DE CORRELACIONES DE FLUJO MULTIFÁSICO VERTICAL}

En 1973, Lawson y Brill (1973) presentaron resultados obtenidos de 6 correlaciones usadas para estimar caídas de presión de flujo multifásico en tuberías verticales, contrastando las correlaciones de: Hagedorn y Brown, Fancher y Brown, Duns y Ros, Poettmann y Carpenter, Baxendell y Thomas, y Orkiszewski. En 1974, Vohra et al (1974), examinaron las correlaciones de Beggs y Brill, Chicerici et al. (1974), y Aziz et al. Los nueve métodos mencionados fueron evaluados implementando un banco de datos consistente de 726 casos de pozos, por medio del cálculo de la diferencia porcentual promedio y de la desviación estándar. Además, el desempeño de cada modelo de flujo fue evaluado dentro de rangos predeterminados de cinco variables: diámetro de la tubería, ${ }^{\circ} \mathrm{API}$, relación gaslíquido de producción, relación agua- petróleo de producción, y, velocidad superficial de la mezcla. Los autores establecieron por medio del menor error porcentual promedio, los tres mejores métodos: i) Hagedorn y Brown,-1.3 [\%]; ii) Francher y Brown, -5.5 [\%]; y, iii) Aziz et al., +8.2 [\%]; y en base a la menor desviación estándar: i) Hagedorn y Brown, 26.1; ii) Beggs y Brill, 36.1; y, iii) Aziz et al., 34.7.

En 1979, Rossland llevó a cabo una investigación en relación al desempeño de las correlaciones de caída de presión para pozos de alta capacidad, la cual fue conducida en 130 pozos direccionales, cuyo rango de operaciones fue: GOR de 480 a 19,000 [SCF/STB], profundidades de pozos de 5,000 a 11,000 [Ft], y diámetros de tubería de 2.6 a 5.1 [in]. Las correlaciones evaluadas por Rossland fueron: Hagedorn y Bronw, Orkiszewski, Duns y Ros, Beggs y Brill, y, Poettmann y Carpenter. El autor determinó que Hagedorn y Brown era la mejor correlación, con base al error porcentual promedio y la desviación estándar obtenidos; (-3.5 y 8.5 , respectivamente). También demostró la factibilidad de aplicar a pozos direccionales, correlaciones que inicialmente habían sido desarrolladas para pozos verticales.

Asimismo, durante este año, Ibe (1979) presentó los resultados obtenidos de un estudio comparativo de las correlaciones de: Hagedorn y Brown, Orkiszewski, Duns y Ros, y Beggs y Brill. En este estudio, se evaluaron 892 casos de pozos, de los cuales, 300 eran pozos direccionales, cuyo rango de GOR oscilaba entre 0 y 78,800 [SCF/STB], con profundidades entre 1000 a 1200 [Ft], y diámetros de tubería entre 1 a 8.8 [in]. La correlación de Orkiszewski mostró un mejor desempeño en cuanto al error porcentual promedio (-0.75 [\%]), seguida de la correlación de Hagedorn y Brown, +1.24 [\%]; siendo esta última la de menor desviación estándar, 23.3.

En 1994 Ansari et al, presentaron en su estudio la formulación de un modelo mecanístico para flujo bifásico. El modelo fue evaluado con un extenso rango de información experimental y de campo obtenido de la Universidad de Tulsa. El desempeño del modelo fue comparado con 6 correlaciones empíricas (i.e. Hagedorn y Brown, Duns y Ros, Orkiszewaki, Beggs y Brill, Mukherje y Brill, Aziz et al.) y un modelo mecanístico (i.e. Hasan y Kabir). La comparación fue realizada por medio del cálculo del factor de desempeño relativo, definido por los autores a través de relaciones de parámetros estadísticos de error. La correlación de Hagedorn y Brown es postulada como la más apropiada para adaptarse a diversos tipos de pozo y regímenes de flujo (i.e. Pozos verticales, Pozos desviados, pozos con $100[\%]$ flujo tapón).

\section{COMPARACIÓN DE CORRELACIONES DE FLUJO MULTIFÁSICO HORIZONTAL}

Mandhane et al. $(1974,1976)$ llevaron a cabo un estudio comparativo de los métodos de predicción de holdup de líquido y factor de fricción para flujo multifásico horizontal. Implementaron una base de datos de 2,685 puntos de mediciones de holdup de líquido y más de 10,000 puntos de caída de presión. Para el primer enfoque evaluaron 8 correlaciones (i.e. Beggs y Brill, Dukler et al., Baroczy (1966), Chenoweth y Martin (1655), Hoogendorn (1959), Chawla (1969), Lockhart y Martinelli, Chisholm (1969)), y para el segundo 10 (i.e. incluyeron las correlaciones de Bertuzzi et al. (1956) y Baker (1954)). Los datos fueron clasificados de acuerdo al patrón de flujo evidenciado, según 
al mapa de patrones propuesto por los autores. Se calcularon errores porcentuales promedio, y desviaciones estándar, resultando la correlación de Beggs y Brill con una mayor precisión (i.e. errores porcentuales de 10.4 [\%], y, -4.7 [\%]; respectivamente para cada estudio).

Gregory et al. (1974) presentaron un modelo híbrido para la estimación de pérdidas de presión, el cual fue comparado con tres métodos (i.e. Beggs y Brill, Dukler y Eaton, Flanigan). Los autores utilizaron un banco de información de 2,685 puntos de holdup de líquido, y 10,000 mediciones de caída de presión, sometidas a efectos de elevación provenientes del terreno (i.e. gradiente de presión- componente de elevación). La correlación de Beggs y Brill es identificada como el modelo que provee mejores estimaciones, por medio del error porcentual promedio, y la desviación estándar (i.e. 2.0 [\%], y 17.3; respectivamente), seguida del modelo híbrido propuesto por los autores (i.e. -3.1 [\%], y, 11.7; respectivamente).

Vohra et al. (1975) compararon el desempeño de correlaciones de flujo multifásico, a través de la estimación del: i) factor de fricción, y, ii) holdup de líquido. Para el primer estudio, usaron valores de holdup de líquidos medidos, con el objeto de contrastar 4 correlaciones (i.e. Beggs y Brill, Duklert, Guzhov (1967), Eaton et al.) Los factores de fricción calculados fueron evaluados a través del error porcentual promedio y la desviación estándar, resultando la correlación de Beggs y Brill, la de mejor desempeño (-12.0, y 31.4; respectivamente), seguida de la correlación de Dukler (-9.4, y, 32.4; respectivamente). Para el segundo enfoque, evaluaron 6 correlaciones (i.e. Beggs y Bril, Dukler, Eaton et al., Guzhov, Hughmark (1962), Lockhart y Martinelli, No-slip). Para la cual, se obtuvieron resultados similares, siendo la correlación de Beggs y Brill la de menor error porcentual, 6.0 [\%], y de desviación estándar, 17.2.

Cabe mencionar que el banco de mediciones utilizados para los estudios relacionados con flujo vertical, no contaba con suficientes datos de campo para realizar una evaluación imparcial, debido a que implementaban los mismos usados para la formulación de las correlaciones de Beggs y Brill, Dukler, y, Eaton et al. Posteriormente, aparecieron los estudios de Fayed y Otten (1983), Osman y El-Feky (1985), y, Asheim (1986), los cuales evaluaron las correlaciones con una mayor cantidad de información, siendo la correlación de Beggs y Brill la de mejor desempeño.

\section{CARACTERZACION FISÍCA DEL FLUIDO}

Los procesos involucrados en los cálculos ingenieriles de yacimiento requieren un buen conocimiento de las propiedades físicas de los fluidos. Tales propiedades representan una importante herramienta en la predicción del desempeño del yacimiento. Dentro de estos cálculos se incluyen: balance de masa, estimación de reservas, diseño y operaciones de producción. Dos métodos son ampliamente implementados para su determinación: i) Ecuaciones de Estado, y ii) Correlaciones PVT. Las ecuaciones de estado están basadas en conocer en detalle la composición de los fluidos. No obstante, tales datos no siempre están disponibles, debido principalmente a los costos y tiempo derivado de estos procesos. Por otro lado, las correlaciones PVT son determinadas a partir de un conjunto de muestras obtenidas desde fondo de pozo o superficie, y están basadas en mediciones fáciles de obtener en campo: presión y temperatura de yacimiento, gravedad específica del aceite y el gas (Valle, et al., 2017).

En la literatura hay muchas correlaciones para la predicción de diferentes propiedades PVT, resultando en un extenso volumen de publicaciones; las cuales han sido desarrolladas usando técnicas de regresión multivariable lineales o no lineales. Existen un gran número de gráficas y correlaciones matemáticas para determinar la presión de burbuja, la relación gasaceite, las viscosidades y factores volumétricos de los fluidos. En la Tabla 2, se evidencian los rangos de implementación de algunos modelos.

\section{MODELO TÉRMICO}

A medida que el fluido se mueve a través de la tubería, éste constantemente intercambia calor con el entorno, resultando por lo general, en una declinación de la temperatura del mismo. El perfil de temperatura del fluido es afectado por los cambios de elevación y de velocidades, por la transferencia de calor, y por el efecto Joule-Thompson (Gould, 1979). El problema de la transferencia de calor en tuberías ha sido ampliamente investigado. Diferentes modelos han sido propuestos y utilizados dependiendo de: i) las condiciones del entorno (i.e. tubería aireada- Ortiz y Jaimes, 2015, tubería parcialmente y completamente enterradaChukwuemeka, 2010, Woom et al., 2014, Arthur et al., 2016-); ii) la geometría de la tubería (i.e. vertical -González y González, 2013-, horizontal -Romero y Salazar, 2007- ); y, iii) los patrones de flujos (e.g. Zhang, 
et al., 2006). Su dificultad radica en el cálculo del coeficiente global de transferencia de calor (U), el cual, unifica los fenómenos involucrados en el proceso (i.e. conducción, convección, radiación). En la Ecuación 1, se expresa el flujo de calor:

$$
Q=U A \Delta T
$$

Donde Q es la transferencia de calor, BTU/h; $U$ es el coeficiente global de transferencia de calor, BTU/h $\mathrm{ft}^{2 \circ} \mathrm{F}, A$ es el área transversal, $\mathrm{ft}^{2}, \mathrm{y} \Delta \mathrm{T}$ es la diferencia de temperatura entre dos resistencias, ${ }^{\circ} \mathrm{F}$.

Dependiendo del número de resistencias por la cual circule el calor, mayor será la complejidad del cálculo de U. Es de gran importancia considerar las diferentes conductividades térmicas, exhibidas por los materiales de elaboración de las tuberías, y sus recubrimientos/ aislamientos. En las Tabla 3 y Tabla 4, se ofrecen algunas conductividades térmicas. Asimismo, se deben detallar las condiciones del entorno, por lo cual, en la Tabla 5 se evidencian algunas conductividades térmicas de materiales sólidos.

\section{PRINCIPIOS DE SIMULACIÓN}

Los programas de simulación en estado estable existentes (Ver Tabla 1) se fundamentan en la ecuación de balance de energía mecánica o gradiente de presión, para la estimación de parámetros hidráulicos (i.e. velocidades, caudales, presiones), constituido de 3 componentes: i) Fricción; ii) Elevación; y iii) Aceleración (Gregory, et al., 1974). En la ecuación 2, se observa este comportamiento.

Tabla 2. Rango de aplicación de correlaciones PVT. Modificado de (Bergman y Sutton, 2009; Moradi, et al., 2010)

\begin{tabular}{|c|c|c|c|c|c|c|c|}
\hline \multirow{2}{*}{ Modelo PVT } & \multicolumn{7}{|c|}{ Rango de Aplicación } \\
\hline & $\mathrm{Pb}[\mathrm{Psi}]$ & $\begin{array}{c}\text { Rs } \\
\text { [SCF/STB] }\end{array}$ & Bo [BBI/STB] & API & $\mathbf{T}\left[{ }^{\circ} \mathbf{F}\right]$ & $\square \mathbf{g}$ & $\begin{array}{c}\text { Viscosidad Aceite } \\
\text { Muerto [cp] }\end{array}$ \\
\hline Standing & $130-7000$ & $20-1425$ & $1.0240-2.150$ & $16.5-63.8$ & $100-258$ & $0.59-0.95$ & $0.865-1550$ \\
\hline Vazquez-Beggs & $15-6055$ & $0-2199$ & $1.028-2.226$ & $15.3-59.3$ & $75-294$ & $0.65-1.28$ & - \\
\hline Glaso & $165-7142$ & $90-2637$ & $1.032-2.588$ & $22.3-48.1$ & $80-280$ & $0.65-1.276$ & - \\
\hline Al-Marhoun & $130-3573$ & $26-1602$ & $1.032-1.997$ & $19.4-44.6$ & $74-240$ & $0.75-1.367$ & - \\
\hline Petrosky-Farshad & $1574-6528$ & $217-1406$ & $1.118-1.623$ & $25.4-46.1$ & $114-288$ & $0.58-0.85$ & $0.725-10.25$ \\
\hline De Ghetto et al. & $107.33-6613.82$ & $8.61-3298.66$ & $1.034-2.887$ & $6-56.8$ & $81-342$ & $0.624-1.789$ & $0.46-1386.9$ \\
\hline Kartoatmodjo & $24.7-4746.7$ & $0-2890$ & $1.007-2.747$ & $14.5-59$ & $80-320$ & $0.4824-1.1668$ & $0.506-682$ \\
\hline Chew y Conally & $132-5645$ & $51-3544$ & - & - & $72-292$ & - & $0.377-50$ \\
\hline Elsharkawy & - & $10-3600$ & - & $12.3-22.1$ & - & - & $0.005-20.8$ \\
\hline Hossian & $22-1458$ & $19-493$ & & $15-21$ & $51-93.3$ & - & $3.6-360$ \\
\hline Lasater & $48-5780$ & 3-12905 & - & $17.9-51.1$ & $82-272$ & $0.574-1.223$ & - \\
\hline
\end{tabular}

Tabla 3. Conductividades térmicas de distintos materiales para elaboración de tubería. Modificado de (Bratland, 2008)

\begin{tabular}{|cc|}
\hline Material de Elaboración de la Tubería & $\mathbf{K j}\left[\mathbf{B T U} / \mathbf{h} \mathbf{f t}^{\circ} \mathbf{F}\right]$ \\
\hline Acero & 26 \\
\hline Hierro Dúctil & $26-30$ \\
\hline Aluminio & 115.53 \\
\hline Cobre & 219.56 \\
\hline Polietileno de alta densidad & 0.2080 \\
\hline PVC & 0.10978 \\
\hline Concreto & $0.491-0.99$ \\
\hline Fibra de vidrio & 0.10169 \\
\hline
\end{tabular}

Tabla 4. Conductividades térmicas de distintos materiales para revestimiento/aislamiento de tubería. Modificado de (Bratland, 2008)

\begin{tabular}{|cc}
\hline Material recubrimiento/aislante & $\mathbf{K j}\left[\mathbf{B T U} / \mathbf{h} \mathbf{~ f t}{ }^{\circ} \mathbf{F}\right]$ \\
\hline Asfalto & $9.8224 \mathrm{e}-2$ \\
Cemento & 0.60668 \\
Concreto & $5.7779 \mathrm{e}-2-0.34667$ \\
\hline Fusión Epoxi & 0.14503 \\
\hline Fibra de vidrio & $2.3112 \mathrm{e}-2$ \\
\hline Poliestireno & $9.0713 \mathrm{e}-2$ \\
\hline Espuma de Poliestireno & $1.675 \mathrm{e}-2-2.0223 \mathrm{e}-2$ \\
\hline PVC & 0.10978 \\
\hline Espuma PVC & $2.3112 \mathrm{e}-2$ \\
\hline
\end{tabular}


Tabla 5. Conductividades térmicas de materiales sólidos. Modificado de (Bratland, 2008)

\begin{tabular}{cc}
\hline Material solido-entorno & $\mathbf{K j}\left[\mathbf{B T U} / \mathbf{h} \mathbf{f t}^{\circ} \mathbf{F}\right]$ \\
\hline Peat (seca) & $9.8224 \mathrm{e}-2$ \\
\hline Peat (humeda) & 0.31201 \\
\hline Suelo Arenoso (seco) & $0.2311-0.4044$ \\
Suelo Arenoso (húmedo) & $0.4911-0.6066$ \\
Arcilla (seca) & $0.35-0.288$ \\
Arcilla(humeda) & $0.4044-0.491$ \\
Caliza & 0.7511 \\
Arenisca & $0.941-1.201$ \\
\hline$\frac{d P}{d L}=\left(\frac{d P}{d L}\right)_{f}+\left(\frac{d P}{d L}\right)_{E l}+\left(\frac{d P}{d L}\right)_{\text {Ace }}$
\end{tabular}

Por lo tanto, para el uso correcto de cualquier simulador hidráulico, se propone considerar 4 modelos para el ajuste a las condiciones reales: modelo físico, modelo de Fluidos, modelo de transferencia de calor, y, modelo de correlaciones de flujo multifásico. En la Figura 3 se presentan los requerimientos para la construcción de los 3 primeros modelos, en función de los parámetros que afectan cada componente del gradiente de presión.

A continuación se listan unas pautas para el modelado de redes de recolección, atendiendo los modelos propuestos:

1. Modelo de fluidos: Dos enfoques se han propuesto para simular la transferencia de masa en sistemas de hidrocarburo: el modelo de composición constante o aceite negro (Black oil) y el modelo composicional (composición variable). De esta manera, de acuerdo al tipo de fluido y a la disposición de información para el modelado del mismo, se recomienda que para aceites volátiles y gas condensado, se implemente un modelo composicional, sin embargo es menester conocer una composición detallada del fluido. Si se cuenta con una pobre descripción de la composición del fluido, se recomienda utilizar el enfoque black oil, considerando que no existirá transferencia de masa entre las fases, por lo cual se emplea para aceites negros. Para este último, es necesario conocer información como: presión de burbuja, ${ }^{\circ}$ API, gravedad específica del gas, viscosidad del aceite, relación gas-aceite, factor volumétrico del aceite, temperatura de yacimiento. Es importante seleccionar alguna de las correlaciones de fluidos provistas por el simulador a partir de un screening, con el objeto de determinar la correlación que mejor se adapte a las condiciones físicas del fluido estudiado (Ver Tabla 2).

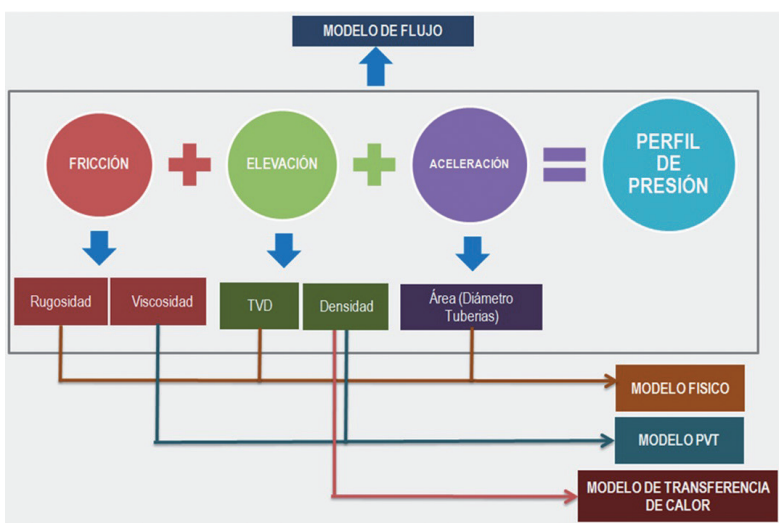

Figura 3. Requerimientos generales para modelar flujo multifásico

2. Modelo Físico: Lo definen las características topográficas del terreno, las particularidades de la tubería (i.e. diámetros, espesores, rugosidad-por lo general se asume de 0.0018 [in]-, tipo de material), y los componentes de la red (i.e. números de pozos, válvulas, choques, entre otros).

3. Modelo de transferencia de calor: Se especifican las condiciones ambientales y características de la tubería tanto externa como interna. Por lo tanto se deben conocer: el diámetro de la tubería, la profundidad de enterramiento (si cumple), la temperatura ambiente, el diámetro del recubrimiento (si cumple), la conductividad del recubrimiento (si cumple), la velocidad del viento (si cumple, tubería aireada), la conductividad del material solido del entorno (si cumple, tubería enterrada). Se recomienda que el cálculo global de transferencia de calor, factor que requieren los simuladores, se realice por medio de una herramienta especializada en procesos.

4. Modelo de correlaciones de flujo multifásico: Una vez calibrado los anteriores modelos, es necesario seleccionar la correlación apropiada de flujo. Anteriormente, se sugirió según estudios literarios, la implementación de la correlación de Beggs y Brill, para flujo horizontal, y la de Hagedorn y Brown, para flujo vertical. Estas pueden dar una aproximación rápida sin realizar un mayor esfuerzo, sin embargo, se recomienda establecer un estudio comparativo de resultados, a través de las correlaciones disponibles en la herramienta a utilizar. 
Por lo tanto, es necesario implementar métodos estadísticos robustos de ponderación de error $\mathrm{y}$ desempeño, como la metodología desarrollada por Ansari et al. (1994), o realizar un sencillo contraste por medio del error absoluto promedio. Se aconseja que los modelos sean ajustados con datos de salida de presiones (resultados), por lo cual, el usuario deberá ingresar caudales.

\section{CONCLUSIONES}

La revisión de diferentes modelos involucrados en la caracterización del flujo multifásico es mostrada, desde modelos de correlaciones de flujo multifásico, hasta modelos de fluidos.

Se propone una metodología para el manejo de cualquier simulador en estado estable de flujo multifásico, consistente en el establecimiento de 4 modelos: Modelo físico, modelo de Fluidos, modelo de Transferencia de Calor, y, modelo de correlaciones de Flujo multifásico.

\section{AGRADECIMEINTOS}

Los autores agradecen a la Universidad Industrial de Santander, al grupo de Modelamiento de Procesos de Hidrocarburos y a Petroleum Consulting Company S.A.S.

\section{REFERENCIAS}

1. Ahmed, M. y Mohammed, A., (2014). A comprehesive Study on the current Pressure Drop Calculation in Multiphase Vertical Wells; Current trends and Future Prospective. s.1.:Journal of Applied Sciences, vol 23, pp 3162-3171, doi: 10.3923/ jas.2014.3162.3171.

2. Ansari, A. y otros, (1994). A Comprehensive Mechanistic Model for Upward Two-Phase Flow in Wellbores. s.1.:SPE Prod. Facil. J., 9 (2), 143-151.

3. Arthur, C., Russell, L. y Adames, P., (2016). An Investigation of Buried Pipe Outer Heat Transfer Coefficient Correlatons. s.1.:BHR Group, 2016179 BHR.

4. Asheim, H., (1986). MONA, an Accurate Two-Phase Well Flow Model Based on Phase Slippage. s.1.:Society of Petroleum Engineers, SPE.

5. Aziz, K., Govier, G. y Forgarasi, M., (1972). Pressure Drop in Wells Producing Oil and Gas. s.1.:J. Can. Pet. Technol., 11 (3), 38-48.
6. Baker, O., (1954). Design of Pipelines for Simultaneous Flow of Oil and Gas. s.1.:OGJ. V. 53.

7. Baroczy, C., (1966). Chem.Eng. Prog. s.1.:s.n.

8. Baxendell, P. y Thomas, R., (1961). The Calculation of Pressure Gradients In High-Rate Flowing Wells. s.1.:J. Pet. Technol., 13 (10), 1023-1028.

9. Beggs, H. y Brill, J., (1973). A Study of Two-Phase Flow in Inclined Pipes. s.1.:J. Pet. Technol., Trans., AIME, 25 (5), 607-617.

10. Bendiksen, K., Malnes, D., Moe, R. y Nuland, S., (1991). The Dynamic Two-Fluid Model OLGA: Theory and Application. s.1.:SPE Prod. Eng, 6 (2), 171-180 (SPE Paper 19451)..

11. Bergman, D. y Sutton, R., (2009). A Consistent and Accurate Dead-Oil-Viscosity Method. s.1.:Society of Petroleum Engineer, SPE 110194-PA.

12. Bertuzzi, A., Tek, M. y Poettman, F., (1956). Simultaneous Flow of Liquid and Gas Through Horizontal Pipe. s.1.:Society of Petroleum Engineers, SPE 544-G.

13. Bratland, O., (2008). Update on commercially available flow assurance software tools: What they can and cannot do and how reliable they are. Kuala Lumpur: 4th Asian Pipeline Conference \& Exposition 2008.

14. Brill, J. y Arirachakaran, S., (1992). State-of-the-Art in Multiphase Flow. s.1.:J.Pet. Technol., 538541, Society of Petroleum Engineers, SPE, No. 23835.

15. Chawla, J., (1969). Liquid Content in Pipes in TwoPhase Flow of Gas-Liquid Mixtures. s.1.:Chimie Ingenieur Technik,69.

16. Chenoweth, J. y Martin, M., (1955). Turbulent TwoPhase FLow. s.1.:Petr.Ref.

17. Chierici, G., Ciucci, G. y Sclocchi, G., (1974). TwoPhase Flow in Oil Wells-Prediction of Pressure Drop. s.1.:J.Pet.Tech. pp 927-937; Trans., AIME, 257.

18. Chisholm, D., (1967). A Theoretical Basis for the Lockhart-Martinelli Correlation for Two-Phase Flow. s.l.:Int. J. Heat and Mass Transfer, 10. 
19. Chukwuemeka, O., (2010). Steady-State Heat Transfer Models For Fully and Partially Buried Pipelines. s.1.:Society of Petroleum Engineers, SPE 131137.

20. Danielson, T., Bansal, K., Hansen, R. y Leporcher, E., (2005). LEDA: The Next Multiphase Flow Performance Simulator. s.l.:In Proceedings of the 9th International Conference on Multiphase Technology, Barcelona, Spain; BHR Group Limited: Cranfield, Bedfordshire,U.K., pp 477-492.

21. Darcy, H., (1857). Recherches Experimentales Relatives au Mouvement de L'Eau dans les Tuyaux. s.1.:Vol. 2; Mallet-Bachelier: Paris, $268 \mathrm{pp}$.

22. Di Lullo, A., (2012). Discover a Career: Flow Assurance. s.1.:Society of Petroleum Engineer, SPE 0112024-TWA.

23. Dukler, A., (1969). Gas Liquid Flow in Pipelines: I. Research Results. s.1.:AGA-API Project NX-28.

24. Duns, H. y Ros, N., (1963). Vertical Flow of Gas and Liquid Mixtures in Wells. s.l.:In Proceedings of the Sixth World Petroleum Congress, Frankfurt, Germany; pp 451-465 (Section II, Paper 212. PD6).

25. Eaton, B., Andrews, D., Knowles, C. y Brwon, K., (1967). The Prediction of Flow Patterns, Liquid Holdup and Pressure Losses Occurring During Continuous Two-Phase Flow in Horizontal Pipelines. s.1.:J. Pet. Technol, 19 (6), 815-828 (SPE Paper 1525).

26. Ellul, I., Saether, G. y Shippen, M., (2004). The Modeling of Multiphase Systems Under Steady State and Trasient Conditions. s.l.:In Proceedings of the Pipeline Simulation Interest Group (PSIG), 36th Annual Meeting,Palm Springs, CA.

27. Fayed, A. y Otten, L., (1983). Comparing Measured with Calculated Multiphase Flow Pressure Drop. s.l.:PennWell Publishing Corp., p 136140.

28. Fernandez, R., Semiat, R. y Dukler, A., (1983). Hydrodynamic Model For Gas- Liquid Slug Flog in Vertical Tubes. s.1.:AIChE J, vol. 29, pp. 981-989.
29. Flanigan, O., (1958). Effect of Uphill Flow on Pressure Drop in Design of Two-Phase Gathering Systems. s.1.:Oil Gas J.

30. González, C. y González, Y., (2013). Transferencia de Calor en Pozos Productores de Hidrocarburos. s.1.:Tesis de Grado. Universidad Nacional Autónoma de México.

31. Gould, T., (1979). Compositional Two-Phase Flow in Pipelines. s.1.:Society of Petroleum Engineers, SPE 5685.

32. Gray, H., (1974). Subsurface Controlled Safety Valve Sizing Computer Program, Appendix B. In Vertical Flow Correlation in Gas Wells, User Manual for API 14BM. s.1.:API: Dallas, TX.

33. Gregory, G., Mandhane, J. y Aziz, K., (1974). Some Design COnsiderations of Two-Phase Flow in Pipes. s.1.:Petroleum Society of Canada, PETSOC 750107.

34. Guzhov, A., (1967). A Study of Trnsprtation in Gas -Liquid Systems. s.1.:10 th INt. Gas Conf., Hamburg-Germany.

35. Hagedorn, A. y Brown, K., (1965). Experimental Study of Pressure Gradients Occurring During Continuous Two-Phase Flow in SmallDiameter Vertical Conduits. s.1.:J. Pet. Technol, 17 (4), 475-484.

36. Hasan, A. y Kabir, S., (1988). A Study of Multiphase Flow Behavior in Vertical Wells. s.l.:SPE Prod. Eng., 3 (2), 263-272 (SPE Paper 15138).

37. Holmes, J., (1977). Description of the Drift Flux Model in the LOCA Code Relap-UK. s.l.:Inst. Mech. End. 103-108 (Paper No. C206/77).

38. Hoogendorn, C., (1959). Gas-Liquid Flow in Horizontal Pipes. s.1.:Chem. ENg. Sci., 9.

39. Hughmark, G., (1962). Holdup in Gas-Liquid Flow. s.1.:Chem. Eng. Prog. 68.

40. Jerez-Carrizales, M., Jaramillo, J. y Fuentes, D., (2015). Prediction of Multiphase Flow in Pipelines: Literature Review. s.l.:Ingenieria y Ciencia, vol. 11, No. 22, pp. 213-233, doi:10.17230/ingciencia.11.22.10.

41. Lawson, J. y Brill, J., (1973). A statistical Evaluation 
of Methods used to Predict Pressure Losses for Multiphase FLow in Vertical Oil Well Tubing. s.1.:Society of Petroleum Engineers, SPE 4267.

42. Lockhart, R. y Martinelli, R., (1949). Proposed Correlation of Data for Isothermal Two-Phase, Two-Component Flow in Pipes. s.1.:Chem. Eng. Progress, 45 (1), 39-48.

43. Mandhane, J., Gregory, G. y Aziz, K., (1974). Critical Evaluation of Holdup Prediction Methods for Gas-Liquid Flow in Horinzontal Pipes. s.1.:Society of Petroleum Engineers, SPE 5140.

44. Mandhane, J., Gregory, G. y Aziz, K., (1976). Critical Evaluation of Friction Pressure Drop Prediction Methods for Gas-Liquid Flow in Horizontal Pipes. s.1.:Society of Petroleum Engineers, SPE 6036.

45. Moody, L., (1944). Friction Factors for Pipe Flow. s.1.:Trans. ASME 66 (8), 671-684.

46. Moradi, B. y otros, (2010). Buble point Pressure Empirical Correlation. s.1.:Society of Petroleum Engineers, SPE 132756-MS.

47. Mukherjee, H. y Brill, J., (1985). Pressure Drop Correlations for Inclined Two-Phase Flow. s.1.:J. Energy Resources Technol., Trans., ASME; 107, (December), 549-468.

48. Orkiszewski, J., (1967). Predicting Two-Phase Pressure Drops in Vertical Pipes. s.1.:J. Pet. Technol., Trans. AIME, 19 (6), 829-838 (SPE Paper 1546-PA).

49. Ortiz, G. y Jaimes, K., (2015). Desarrollo de un Software para el Modelamiento del Flujo Multifásico en Tubería Vertical y Horizontal. s.l.:Tesis de Grado. Escuela de Ingeniería de Petróleos.Universidad Industrial de Santander.

50. Osman y El-Feky, (1985). Design Methods for Two-Phase Pipelines Compared, Evaluated. s.1.:OGJ.

51. Petalas, N. y Aziz, K., (2000). A Mechanistic Model for Multiphase Flow in Pipes. s.l.:J. Can. Pet. Technol., 39 (6), 43-55.

52. Poettmann, F. y Carpenter, P., (1952). Multiphase
Flow of Gas, Oil and Water Through Vertical Flow Strings. s.1.:Drill. Prod. Pract., pp 257.

53. Pressman, R., (2009). Software engineering: A Practitioner's Approach. s.1.:McGraw-Hill. 7th Edition.

54. Reynolds, O., (1883). An Experimental INvestigation of the Circum-stances Which Determine Wheter the motion of Water Shall Be Direct or Sinuous, and of the Law of Resisteance in Parallel Channels. s.1.:Philos. Trans. R. Soc., 174, pp. 935-982.

55. Romero, A. y Salazar, D., (2007). Herramienta Computacional para el Estudio del Comportamiento del Flujo Multifásico con Transferencia de Calor en Líneas de Flujo. s.l.:Tesis de Grado. Universidad Central de Venezuela.

56. Rott, N., (1990). Note on the History of the Reynolds Number. s.1.:Ann. Rev. Fluid Mech, pp. 1-11.

57. Shi, H. y otros, (2005). Drift-Flux Modeling of TwoPhase Flow in Wellbores. s.1.:Society Petrleoum Engineers, SPE 84228-PA, 10 (1), 24-33.

58. Shippen, M. y Bailey, W., (2012). Steady-State Multiphase Flow-Past, Present, and Future, with a Perspective on Flow Assurance. s.1.:American Chemical Society, ACS.

59. Shoham, O., (2006). Mechanistic Modeling of GasLiquid Two-Pahse Flow in Pipes. s.1.:Society of Petroleum Engineers, SPE, 408 pp.

60. Taitel, Y. y Dukkler, A., (1976). A Model for Predicting Flow Regime Transitions in Horizontal and Near Horizontal Gas-Liquid Flow. s.1.:AIChE J., 22 (1), 47-55.

61. Valle, G., Romero, F., Mendoza, L. y Osorio, D., (2017). Empirical PVT Correlations Applied For Colombian Crude Oils: A New Approach. s.1.:Society of Petroleum Engineers, SPE 185565.

62. Vohra, I., Hernandez, F., Marcano, N. y Brill, J., (1975). Comparison of Liquid Holdup and Friction Factor Correlations for Gas-Liquid Flow in Horizontal Pipes. s.l.:Society of Petroleum Engineers, SPE 4690. 
63. Vohra, I., Robinson, J. y Brill, J., (1974). Evaluation of Three New Methods For Predicting Pressure Losses In Vertical Oilwell Tubing. s.1.:Society of Petroleum Engineers, SPE 4689.

64. Weisbach, J., (1845). Lehrbuch der Ingenieur- und Maschinen-Mechanik Vol. 1 Theoretische Mechanik. s.1.:Vieweg und Sohn: Braunschwieg, Germany, 535 pp.

65. Wook, D. y otros, (2014). Effect of Buried Depth on Steady-State Heat-Transfer Characteristics for Pipeline-Flow Assurance. s.1.:Society of Petroleum Engineers, SPE 166595.

66. Xiao, J., Shoham, O. y Brill, J., (1994). A Comprehensive Mechanistic Model for TwoPhase Flow in Pipelines. s.1.:Presented at the SPE Annual Technical Conference and Exhibition, New Orleans, LA, Sept. 23-25,SPE Paper 20631.
67. Xiao, J.-J. y Shoup, G., (1998). Sizing Wet- Gas Pipelines and Slug Catchers with Steady-State Multiphase Flow Simulations. s.1.:J. Energy Resources Technology, Trans. ASME, vol. 2, pp. 106-111.

68. Zhang, H., Wang, Q. y Brill, J., (2003). A Unified Mechanistic Model for Slug Liquid Holdup and Transition Between Slug and Dispersed Bubble Flows. s.1.: Int. J. Multiphase Flow, 29 (1), 97-107.

69. Zhang, H., Wang, Q., Sarica, C. y Brill, J., (2006). Unified Model of Heat Transfer in Gas/Liquid Pipe Flow. s.1.:Society of Petroleum Engineers, SPE 90459.

70. Zuber, N. y Findlay, J., (1965). Average Volumetric Concentration in Two-Phase Flow Systems. s.1.:J. Heat Transfer, Trans. ASME, Ser. C, 26 (3), 453 - 468.

Recepción: 21 de Febrero de 2017

Aceptación: 29 de Abril de 2017 